\title{
Sensor-Assisted Wi-Fi Indoor Location System for Adapting to Environmental Dynamics
}

\author{
Yi-Chao Chen ${ }^{\mathrm{a}}$, Ji-Rung Chiang ${ }^{\mathrm{a}}$, Hao-hua Chu ${ }^{\mathrm{a}, \mathrm{b}}$, Polly Huang ${ }^{\mathrm{b}, \mathrm{c}}$, and Arvin Wen Tsui ${ }^{\mathrm{d}}$ \\ Department of Computer Science and Information Engineering ${ }^{\mathrm{a}}$ \\ Graduate Institute of Networking and Multimedia ${ }^{b}$ \\ Department of Electrical Engineering ${ }^{\mathrm{C}}$ \\ National Taiwan University \\ Industrial Technology Research Institute ${ }^{d}$ \\ \{b89066, r93102, hchu\}@csie.ntu.edu.tw, phuang@cc.ee.ntu.edu.tw, arvin@itri.org.tw
}

\begin{abstract}
Wi-Fi based indoor location systems have been shown to be both cost-effective and accurate, since they can attain meter-level positioning accuracy by using existing Wi-Fi infrastructure in the environment. However, two major technical challenges persist for current Wi-Fi based location systems, instability in positioning accuracy due to changing environmental dynamics, and the need for manual offline calibration during site survey. To address these two challenges, three environmental factors (people, doors, and humidity) that can interfere with radio signals and cause positioning inaccuracy are identified. Then, we have proposed a sensor-assisted adaptation method that employs RFID sensors and environment sensors to adapt the location systems automatically to the changing environmental dynamics. The proposed adaptation method performs online calibration to build multiple contextaware radio maps under various environmental conditions. Experiments were performed on the sensor-assisted adaptation method. The experimental results show that the proposed adaptive method can avoid adverse reduction in positioning accuracy under changing environmental dynamics.
\end{abstract}

\section{Categories and Subject Descriptors}

J.m [Computer Applications]: Miscellaneous; C.4 [Computer Systems Organization]: Performance of Systems - Performance attributes.

\section{General Terms}

Measurement, Performance, Design, Experimentation.

\section{Keywords}

Indoor location system, adaptive system, sensors, performance evaluation.

\section{INTRODUCTION}

Location is one of the most widely utilized context data in context-aware and ubiquitous computing applications. To support

Permission to make digital or hard copies of all or part of this work for personal or classroom use is granted without fee provided that copies are not made or distributed for profit or commercial advantage and that copies bear this notice and the full citation on the first page. To copy otherwise, or republish, to post on servers or to redistribute to lists, requires prior specific permission and/or a fee.

MSWiM'05, October 10-13, 2005, Montreal, Quebec, Canada.

Copyright 2005 ACM 1-59593-188-0/05/0010...\$5.00 such location-aware applications in the indoor environment, many indoor location systems [18] have been developed in the past decade with different deployment costs and positioning accuracy levels. A promising approach is the Wi-Fi based location estimation system, which is cost-effective by employing existing IEEE 802.11 network infrastructure available in many office and home environments. The proposed approach can provide meterlevel accuracy, which is sufficient for most location-aware applications.

Wi-Fi based location systems generally work in two phases. Phase 1 is called the offline training phase, in which a human operator performs a site survey by measuring the received signal strength indicator (RSSI) from different access points (APs) at some fixed sampled points in the environment. These RSSI measurements are recorded onto a radio map that depicts the RSSI values of APs at different sampled points. Phase 2 is known as the online estimation phase, in which the target's location is calculated in real time by matching sampled points on the radio map with the closest RSSI values to the target.

Current Wi-Fi based location systems have two general problems. The first problem is the amount of manual calibration effort needed to build the radio map during the offline training phase. Users must compile a fairly dense radio map comprising many RSSI measurements at many sampled points to attain reasonable positioning accuracy. For example, the Ekahau location system [13] requires 80 RSSI samples to be taken every 3 meters to attain an average positioning accuracy of 3 meters in a $1000 \mathrm{~m}^{2}$ environment, which translates into approximately two man-hours of calibration effort.

The second problem is the instability in the positioning accuracy due to the changing environmental dynamics. The following three dynamic factors have been observed to change frequently over time in the environment, affecting the positioning accuracy: relative humidity level, people presence and movements, and open/closed doors. These environmental factors can interfere with the radio signal propagation from the APs to the target mobile devices, varying the received RSSI. These dynamic environmental factors can incur location estimation errors in the existing Wi-Fi based location systems that construct and maintain only one static radio map, because this single radio map is calibrated by the environmental condition at the time of site survey. When the environmental condition changes later this static radio map may no longer reflect the expected RSSI values in the environment. 


\subsection{Environmental Dynamics}

To determine the quantitative effects of these dynamic environmental factors, preliminary experiments were performed in a corridor on the $6^{\text {th }}$ floor of our department building shown in Figure 1. The corridor is marked as a shaded green line. Five IEEE $802.11 \mathrm{~b}$ access points (marked as triangles $\boldsymbol{\Delta}$ ) are placed inside five rooms along the corridor.

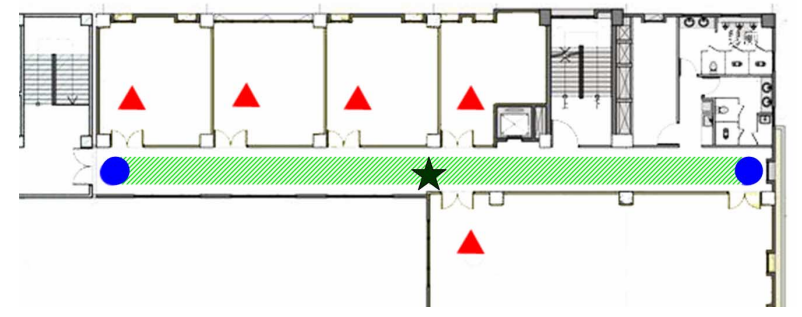

Figure 1. Floor layout for measuring the impacts of environmental factors

- People: The presence of people has a similar effect to obstacles blocking radio signals. The following common people-blocking scenario in a museum was emulated in this experiment. A museum tracks the location of Joe, a visitor, through his mobile device. When Joe stops in front of a popular painting exhibition, other visitors who are interested in that painting exhibition are standing around him. These stand-around visitors are likely to block the radio signals from the APs to Joe's mobile device. To determine how people-blocking impacts RSSI, the following experiment was performed: (1) a formation of six people was arranged surrounding a user carrying the target mobile device - this is called the block-around scenario; (2) a fixed location point (marked as in Figure 1) was chosen on the corridor, and 300 RSSI samples were continuously collected, and then (3) the probability distribution of these RSSI samples was analyzed. The measurement result demonstrates that in the block-around scenario, the RSSI values attenuate rapidly. The average RSSI value is reduced by approximately $8 \mathrm{dBm}$ from the non-blocking scenario to the block-around scenario.

- Doors: open and closed doors have a similar effect to changes in the environment's floor layout. Considering the floor layout in our department building depicted in Figure 1. Since a $\mathrm{Wi}-\mathrm{Fi}$ access point is placed in each room along the corridor, open or closed doors are expected to significantly affect the RSSI received by the target mobile device on the corridor. To determine how doors impact RSSI, the following experiment was performed: (1) 300 RSSI samples were continuously collected in both the open-all-doors and close-all-doors scenarios from the same location point on the corridor, and (2) the probability distribution of these RSSI samples were analyzed. The measurement result demonstrates a significant rise of $9 \mathrm{dBm}$ on the average RSSI from the close-all-doors scenario to the open-all-doors scenario.

- Humidity: the IEEE 802.11 specification adopts a radio frequency of $2.4 \mathrm{GHz}$, which is also the resonant frequency of water. Hence, an environment with a high relative humidity $(\mathrm{RH})$ level tends to absorb more power from the radio signals than in lower RH level. To measure the effect of humidity on the RSSI values, the following experiment was performed: (1) 300 RSSI samples were continuously collected at both the higher RH level $(70 \%)$ and the lower RH level $(40 \%)$ at the same fixed location point on the corridor, and (2) the probability distribution of these RSSI samples were analyzed. The measurement result demonstrates that the average RSSI value falls by $0.8 \mathrm{dBm}$ from $40 \% \mathrm{RH}$ level to $70 \%$ RH level.

Table 1 summarizes how dynamic environmental factors influence the positioning accuracy. The radio map is calibrated under the environmental condition of no-blocking people, close-all-doors, and $40 \%$ relative humidity level, denoted as the baseline radio map. The average positioning accuracy using the ITRI positioning engine [4] is 2.13 meters. This number serves as the baseline for comparison with other scenarios under various environmental conditions. In the people block-around scenario, the average positioning accuracy deteriorates by $85.9 \%$ to 3.96 meters. In the open-all-doors scenario, the average positioning accuracy deteriorates by $236.6 \%$ to 7.17 meters. When the RH level rises to $70 \%$ (e.g., on a raining day), the average positioning accuracy deteriorates by $43.7 \%$ to 3.06 meters. Although the effect of humidity is not as significant as that of people and doors, the change in humidity level still introduces an error of almost one meter to the existing static methods.

Table 1. Average position accuracy under changes in different environmental factors

\begin{tabular}{|c|c|c|c|c|}
\hline \multicolumn{5}{|c|}{$\begin{array}{c}\text { Baseline: Training environmental condition: non-blocking people, } \\
\text { close-all-doors, } 40 \% \text { relative humidity level }\end{array}$} \\
\hline \hline $\begin{array}{c}\text { Environmental } \\
\text { condition }\end{array}$ & $\begin{array}{c}\text { No } \\
\text { change }\end{array}$ & $\begin{array}{c}70 \% \\
\text { humidity }\end{array}$ & $\begin{array}{c}\text { Open- } \\
\text { all-doors }\end{array}$ & $\begin{array}{c}\text { People } \\
\text { block- } \\
\text { around }\end{array}$ \\
\hline Effects & $2.13 m$ & $\begin{array}{c}3.06 m \\
(43.7 \%)\end{array}$ & $\begin{array}{c}7.17 m \\
(236.6 \%)\end{array}$ & $\begin{array}{c}3.96 m \\
(85.9 \%)\end{array}$ \\
\hline
\end{tabular}

\subsection{Sensor-assisted Adaptation}

One naïve effective approach to instable positioning accuracy is to construct and calibrate multiple context-aware radio maps under different environment variations, enabling the system to monitor the current environmental condition, choose the optimally matched radio map to the current state of environmental condition, and use it to estimate the location. Unfortunately, calibrating multiple context-aware radio maps is problematic in two ways: (1) constructing $n$ context-aware radio maps requires repeating the same RSSI sample collection $n$ times, and (2) manipulating environmental conditions, e.g., changing humidity levels in a large facility or assembling various sizes of block-around people at different locations, is non-trivial. These two difficulties make this approach unworkable.

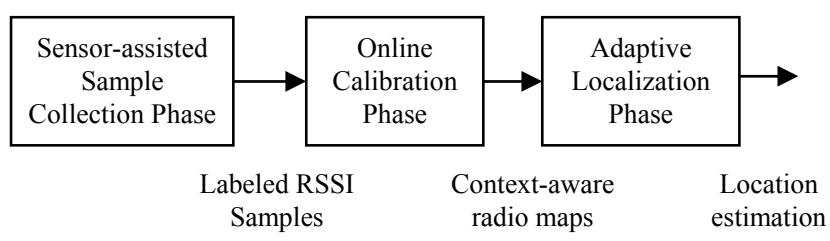

Figure 2. Adaptive Location Positioning System 
To overcome these two difficulties, a solution is proposed to adapt sensors to help generate these context-aware radio maps. The proposed method adopts a subset of RSSI samples obtained over the course of its online usage to automatically train these contextaware radio maps. Our sensor-assisted adaptation comprises three phases depicted in Figure 2 and described below. These phases are different from those (calibration \& estimation) in current Wi-Fi based location systems.

- Sensor-assisted sample collection phase collects RSSI samples and utilizes sensors to label these samples.

- Online calibration phase applies the labeled RSSI samples to train different context-aware radio maps.

- Adaptive localization phase selects the context-aware radio map which best matches the current environmental condition state to estimate the locations of the target mobile devices.

To our knowledge, no known existing Wi-Fi based location systems utilize sensors to effectively overcome environmental dynamics and to enhance the positioning accuracy. This work is believed to be the first to apply sensors to detect variations in the physical environmental factors, apply them to automatically calibrate multiple context-aware radio maps from online samples, and incorporate context-awareness into estimating the Wi-Fi based location.

The remainder of this paper is organized as follows. Section 2 describes related works. Section 3 explains the sensor-assisted adaptation to environmental dynamics. Section 4 presents the experimental results and shows the improvement in positioning accuracy in our approach. Section 5 draws the conclusion and suggests future work.

\section{RELATED WORK}

Many location estimation systems have been developed using WiFi RSSI values to estimate locations. These systems can be categorized into two broad approaches. The first approach is based on the deterministic method [2][4][11][13]. Systems following this approach apply deterministic inference, such as triangulation and $k$-nearest-neighbors (KNN) search, to estimate the target device's location. For example, the RADAR system [4][11] applies KNN to obtain the $k$ nearest neighbors and estimates the location of the target device by averaging the locations of these $\mathrm{k}$ nearest neighbors. The second approach is based on the probabilistic method [10][12][15][16]. Seshadri et al. [10] applied Bayesian's inference, which uses multiple probabilistic models and histograms to enhance the performance of the original system. It calculates the conditional probabilities over locations based RSSI. It also added a motion model to describe the continuity in human's movements such that it can lower the oscillatory location estimations in Wi-Fi based localization systems. Notably, our proposed sensor-assisted adaptation is independent of these two approaches, and can be applied to both approaches and further enhance their positioning accuracy under environmental dynamics.

To reduce the amount of manual calibration effort, several alternative methods that do not require users to collect RSSI samples manually have been proposed. For example, the RADAR system [2] has applied the radio propagation model [8][9][17] to estimate the RSSI at different locations in the environment. The radio propagation model can estimate the level of signal strength fading by analyzing the floor layout, locations and sizes of obstructions, and the attenuation factors associated with these obstacles. Rather than manually measuring RSSI samples in the environments, these systems adopt the radio propagation model to (1) estimate RSSI values for different location points, and (2) compile them into the radio map. However, in practice, the layout of an indoor environment is dynamic. Additionally, the attenuation factors of materials and obstructions are difficult to determine accurately. Moreover, some mobile obstacles can also influence Wi-Fi RSSI from AP to target devices, but their locations are not known in the offline phase. Based on their experimental results, the radio propagation model achieves a lower average positioning accuracy of 4.3 meters than the manual site survey, with 2.94 meters average accuracy. Chai et al. [3] presented another method to lower the amount of user calibration efforts by reducing the quantity of RSSI samples needed during offline site survey. The reduction targets (1) the number of sampled points on the radio map, and (2) the number of RSSI samples gathered at each sampled point. After reducing the number of sampled points, they apply interpolation method to estimate the RSSI values on the missing sampled points. They have reported that by reducing the number of sampled points and samples to $1 / 3$ of the original site survey, the average positioning accuracy is only lowered by $16 \% \sim 6 \%$. Although their proposed system reduces the amount of user calibration effort while preserving system accuracy, it is still considered to be an offline calibration. By comparison, our proposed system is based on online calibration.

Dynamic environmental factors can incur location estimation errors in traditional Wi-Fi location systems. Some proposed methods have attempted to address this issue. The temporal prediction approach in [1] can observe and learn how a radio map changes over time by employing emitters and sniffers to observe the Wi-Fi RSSI variations. By applying regression analysis, the temporal prediction approach can learn the temporal predictive relationship between the RSSI values received by sniffers and those received by the target mobile devices. However, the temporal prediction approach assumes that changes in the environmental factors follow some predictable temporal patterns. Although possibly valid in some environments, this assumption may not apply to many others. For example, the open \& closed doors may be random, depending on the last person entering or leaving the room, and whether she/he tends to open/closed the door behind her/him. The occurrence time and size of blockaround people are difficult to predict in our department, since different numbers of visitors come and go anytime during the day.

Given the readily availability and cost effectiveness of RFID technology, several recent studies [5][6][7][20] have proposed using RFID to track locations. Willis et al. [5] attached passive RFID tags with known locations to the carpet pads, and RFID readers in the shoes to read locations of these passive RFID tags. To reduce the manual efforts of deploying tags, Haehnel et al. [6] used a robot to explore and localize the RFID tags in the space. The LANDMARC system [7] placed active RFID tags on the objects and RFID readers in the environment to track the tags. The GETA Sandals [20] are a footprint-based location system that tracks user locations by embedding ultrasonic sensors and RFID readers inside the sandals. Our proposed method also adopts the RFID technology to help enhance the accuracy of the Wi-Fi location systems. 


\section{SENSOR-ASSISTED INDOOR LOCATION SYSTEM}

The proposed system is based on the following two concepts: (1) it applies sensors to construct context-aware radio maps, and adapts location estimation to environmental dynamics by choosing a radio map that best matches the current environmental condition; (2) it conducts online calibration to automatically gather RSSI samples and to train these context-aware radio maps, saving user efforts. Figure 4 shows the architecture of the proposed system, which consists of the following three phases, sensor-assisted sample collection phase, online calibration phase, and adaptive localization phase, which are described in detail below.

\subsection{Sensor-assisted Sample Collection Phase}

The idea behind the sensor-assisted online sample collection comes from the observation that when a person walks from a starting point to an ending point, his/her movement speed usually remains fairly consistent over the distance traveled known as constant-speed walking. Other cases exist, including stopping in the middle of the path to talk to other people, or hurrying to attend a meeting. In these cases of non-constant-speed walking, the person would complete the distance traveled in a different amount of time from the constant-speed walking. The constant-speed walking cases can be found from the walking distance (e.g., $l$ meters) and the average walking speed of an individual (e.g., $v$ $\mathrm{m} / \mathrm{s})$ by checking whether the time traveled $(t=l / v)$ falls within the range of normal constant-speed walking time. If the individual walks at a constant speed over a distance segment, then the system can accurately approximate the locations of RSSI values obtained on that walking segment from the following observable parameters: time of RSSI collection $\left(t_{i}\right)$, walking velocity $(v)$, starting and ending locations over this walking segment $\left(l_{0}, l_{n}\right)$, and starting and ending times of the walking segment $\left(t_{0}, t_{n}\right)$.

A small number of passive RFID readers [14] with known location coordinates were placed at the specified corners of the corridor to obtain these parameters. Additionally, the target mobile device was attached to a passive RFID tag, enabling it to be read when coming within approximately 2 meters of the passive RFID readers. A person's walking path is divided into multiple walking segments, where each segment is defined as walking from one RFID reader placed at one corner to another RFID reader placed at another corner. The system then observes $\left(t_{0}, t_{i}, t_{n}, l_{0}, l_{n}\right)$ and forwards these parameters to the online RSSI sample filter.

The online RSSI sample filter checks whether the RSSI samples collected over a walking segment are from constant-speed walking. This detection is conducted by checking whether the traveled time over a walking segment falls within the range of constant-speed walking time. Based on a pedestrian walking study [19], we set the constant walking speed range to $1.25 \mathrm{~m} / \mathrm{s}-1.78 \mathrm{~m} / \mathrm{s}$ which captures approximately $70 \%$ of people's constant-speed walking. The fairly conservative range was selected to prevent RSSI samples from non-constant-speed walking from passing through the filter and corrupting the training samples.

After the constant-speed walking RSSI samples are selected, the next step is the RFID-assisted location estimation that approximates the location of these RSSI values. Figure 3 depicts an example of using this method. Two RFID readers are placed in $\left(\mathrm{x}_{0}, \mathrm{y}_{0}\right)$ and $\left(\mathrm{x}_{4}, \mathrm{y}_{4}\right)$. At $\mathrm{t}_{0}$, the user walks past $\left(\mathrm{x}_{0}, \mathrm{y}_{0}\right)$ which denotes the beginning of this walking segment. The user then reaches $\left(\mathrm{x}_{4}, \mathrm{y}_{4}\right)$, which denotes the end of this walking segment, and at time $t_{4} . S S_{1}, S S_{2}$ and $S S_{3}$ denote RSSI values collected at times $t_{1}, t_{2}$, and $t_{3}$ over this walking segment. The position coordinates $\left(x_{i}, y_{i}\right)$, where $i=1-3$, can be estimated from these observable parameters according to the formulas defined below:

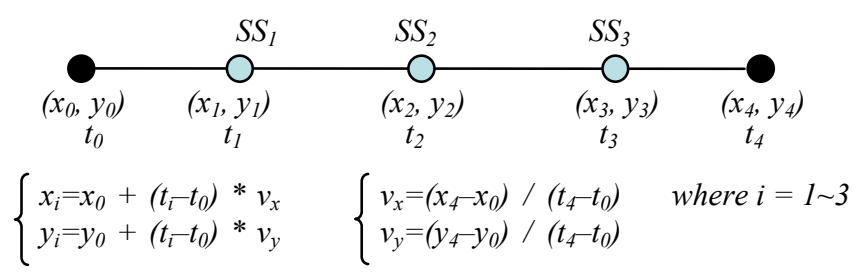

Figure 3. Estimate the location of RSSI samples

Environment sensors were also deployed to monitor the environmental condition state in terms of doors, humidity, and people. Humidity sensors were installed in the environment to detect the current humidity level. The open/closed doors status was obtained by connecting to the RFID/smart card access control systems already installed in most rooms occupied by the department labs. Short-range (10 meters) Bluetooth radio and its RSSI information were used to detect block-around people. People were assumed to carry Bluetooth-enabled mobile devices, since most high-end PDAs and smart phones have Bluetooth radio. The number of people surrounding the user can be estimated from the number of Bluetooth devices around the target user, enabling the block-around condition to be determined.

These RSSI measurements are labeled with (1) locations and (2) environmental condition to calibrate context-aware radio maps as described in the next phase.

\subsection{Online Calibration Phase}

The online calibration phase trains multiple context-aware radio maps from the labeled RSSI samples. One difficulty with online calibration is that collecting enough samples to train an accurate radio map may take several days or weeks. Based on our experiences with our training engine, training an accurate radio map in a $1000 \mathrm{~m}^{2}$ space may need over 200 traces of RSSI samples. The number of traces required is proportional to the size of the environment. When environmental factors are considered, even more RSSI samples are needed to train all possible contextaware radio maps, creating a cold-start problem: the system suffers from poor positioning accuracy during initial deployment before context-aware radio maps have been trained with sufficient samples. This cold-start problem was solved by building a coldstart radio map trained with all RSSI samples from all environmental conditions. If the system cannot find an accurate, context-aware radio map with sufficient training samples, then it refers back to the cold-start radio map to estimate locations.

Consider the following example. The system adapts to the following three environmental factors, people, doors and humidity. Each environmental factor has two possible states. For people, these states are non-blocking or block-around; for doors, they are open or closed, and for humidity, they are high or low. These states combine to give a total of eight possible state combinations corresponding to eight context-aware radio maps. The system initializes eight empty context-aware radio maps and one coldstart radio map. Given a trace of labeled samples, the online 


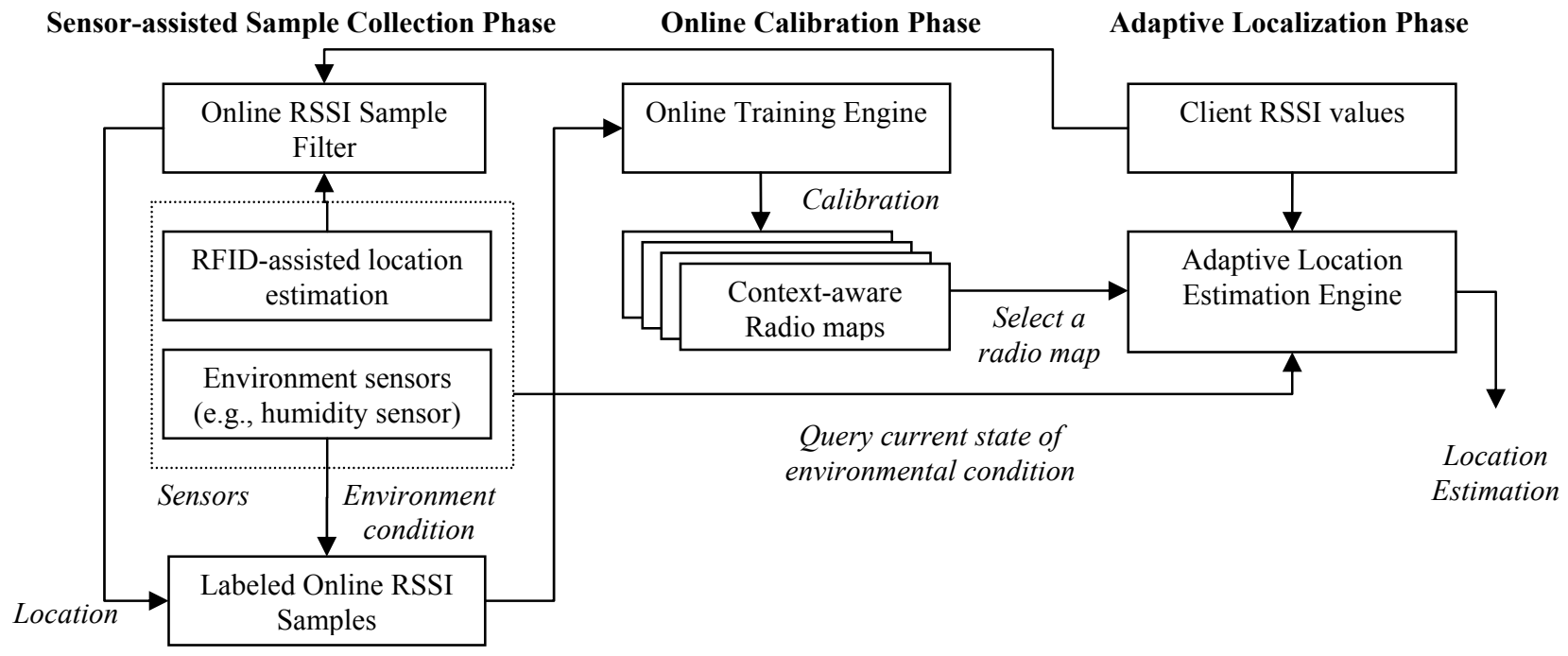

Figure 4. Three phases of our sensor-assisted indoor location system.

training engine looks up the environment label, and trains the corresponding context-aware radio map with the samples. The labeled samples are also applied to train the cold-start radio map.

\subsection{Adaptive Localization Phase}

When the adaptive location estimation engine receives RSSI values from a mobile device, it queries the environment sensors to obtain the current environmental condition, and then choose a radio map that best matches the current environmental condition. If the best-matched radio map has too few training samples (i.e., less than 200 traces of samples), the cold-start radio map is chosen. The chosen radio map can be applied to a location estimation engine to calculate the location of the target.

During the online usage, the adaptive localization phase runs in parallel with the other two phases. When the location system receives RSSI values from a mobile device, both the localization phase and the sample collection phase are executed to run online calibration and online location estimation simultaneously.

\section{EXPERIMENTS}

The following two experiments were performed to evaluate the proposed adaptive indoor location system. In the first experiment, the RFID-assisted online calibration was evaluated based on constant-speed walking and in a static environment state (not considering environmental dynamics). The positioning accuracy of the online and offline calibration was compared. In the second experiment, the adaptive localization that utilizes online calibration to construct context-aware radio maps under changing environmental dynamics was evaluated. The positioning accuracy of adaptive and non-adaptive localization was compared under changing environmental dynamics.

\subsection{Performance Evaluation on RFID-assisted Online Calibration}

To evaluate the performance of our online calibration without being affected by changing environmental factors, the environment state was left unchanged to ensure that only one person walks on the corridor in each time period. Figure 5 shows the layout of this experimental test-bed on the 3rd floor of our department building: the triangles $(\boldsymbol{\Delta})$ mark the locations of APs; the circles $(\bullet)$ mark the locations of RFID readers, and the shaded green lines mark the walking segments.

Three human subjects (graduate students) acted as testers in our experiments. Each subject carried a RFID-tagged PDA and walked along the shaded-lined segments, hitting four RFID readers in both clockwise and counter-clockwise directions. A data trace was denoted by RSSI values received by a subject through a walking segment from one RFID reader to the adjacent reader. A data unit was denoted as RSSI values when a subject walked two circles in the counter-clockwise and clockwise directions. This means that each data unit contains eight data traces. A total of 27 data units (216 data traces) were collected from three human subjects, and all are constant-speed walking. As each data trace is collected online, the system feeds it into the online training engine to refine our radio map, and simultaneously runs the location estimation engine to track the user's position. The results in Figure 6 illustrate that the average positioning accuracy improves as the number of data traces increases, converging to approximately 2.9 meters.

The performance of the manual offline calibration in the traditional Wi-Fi based methods is compared to our automated online calibration. For a fair comparison, the same location estimation engine and site survey software are used to construct the radio map. The site survey selects 40 RSSI samples from each of 24 fixed sampled points separated by 5 meters. Figure 7 shows the experimental setup, where the points mark the sampled locations. The manual offline calibration can achieve an average positioning accuracy of 2.73 meters. By comparison, the offline calibration method achieves similar positioning accuracy to (a slight 0.17 meter better than) our online calibration method. The proposed online calibration method can obtain a similar positioning accuracy without requiring a manual site survey. This advantage becomes significant when considering location systems that can adapt to changing environmental dynamics. When constructing multiple context-aware radio maps, the level of user 


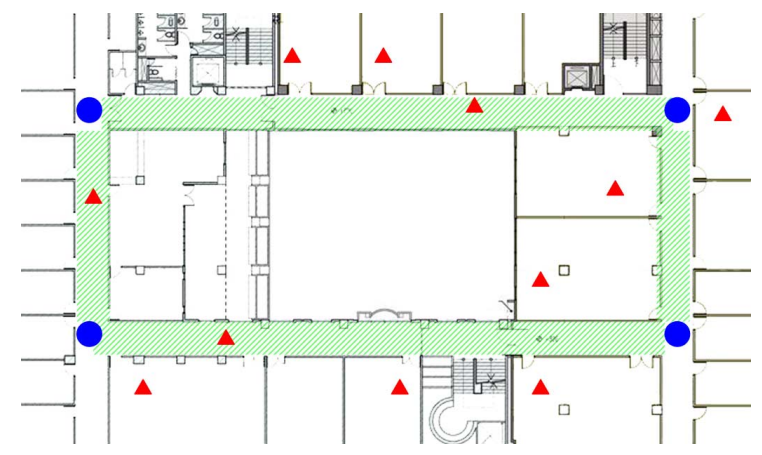

Figure 5. Floor layout for experiment \#1

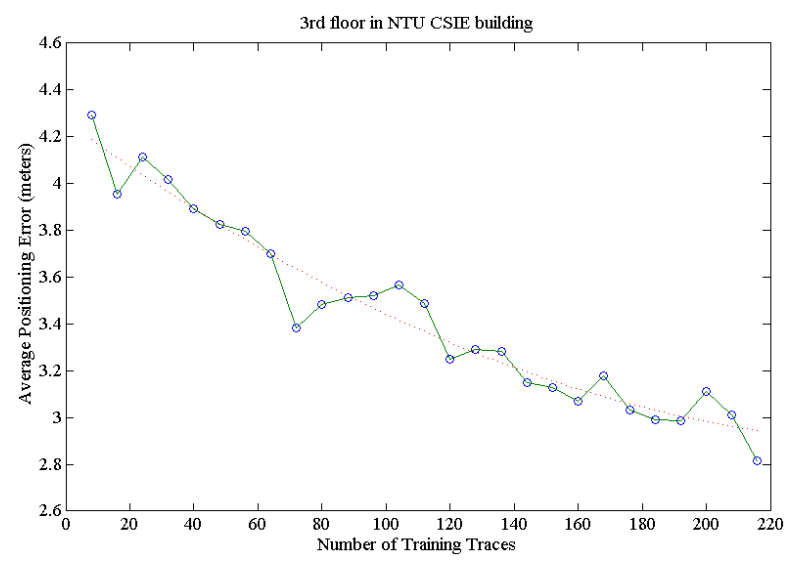

Figure 6. Average positioning accuracy with increasing training traces

effort needed in the offline manual calibration method also multiply, making it impractical.

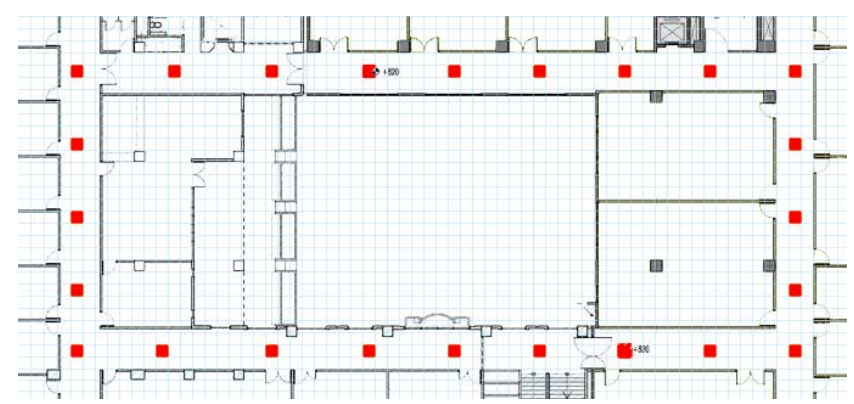

Figure 7. Site survey in the offline training phase. The squares ( $\square$ ) mark the sampled locations

\subsection{Performance Evaluation on Adaptive Localization}

A small area (approximately $400 \mathrm{~m}^{2}$ ) shown in Figure 1 was selected to perform the 2 nd experiment. Since this small area is a closed space, it allows better control and manipulation of the environment state, and then observes how well the adaptive localization adjusts to changing environmental dynamics. This small area had 5 APs depicted as triangles, deployed in 5 different rooms on a corridor. Two RFID readers depicted as circles were deployed at two endpoints of the corridor. The location system tracked a human subject carrying an RFID-tagged PDA and walking along the corridor. Multiple context-aware radio maps were constructed using the proposed online calibration method. To determine how well the proposed location system adapted to different changing environmental factors, one environmental factor state was altered at a time, and change in the positioning accuracy was measured, also showing the effect of each individual environmental factor on the positioning accuracy.

\subsubsection{Impact of Open/Closed Doors}

The experimental setup was described to evaluate how well the proposed adaptive localization adjusted to doors while keeping the other environmental factors (no-blocking people and $40 \% \mathrm{RH}$ level) unchanged. The experiment consisted of the following three steps. In the Step 1, all doors were closed, and online calibration was then applied to train the close-all-doors radio map. In Step 2, all doors were opened, and online calibration was applied to train the open-all-doors radio map. In Step 3, the average positioning accuracy was measured by applying each of two radio maps (close-all-doors \& open-all-doors) to each of the two environmental conditions.

The average positioning accuracy of each of these four mapenvironment combinations is plotted on the left graph of Figure 8 The cumulative distribution functions (CDF) with increasing training samples are plotted on the right graph of Figure 8 . The average positioning accuracy is shown in Table 2 . When the close-all-doors radio map is applied to estimate locations in the same close-all-doors environment, the average positioning accuracy is 2.13 meters after 20 data traces. When the close-alldoors radio map is applied to estimate locations open-all-doors environment, the average positioning accuracy (after 20 data traces) deteriorates by 5.04 meters to 7.17 meters. When the openall-doors radio map is applied to estimate locations in the openall-doors environment, the average positioning accuracy is 2.81 meters after 20 data traces. When the open-all-doors radio map is applied to the close-all-doors environment, the average positioning accuracy (after 20 data traces) deteriorates by 1.78 meters to 4.59 meters. Notably, under changing environmental dynamics, the proposed adaptive localization can avoid applying the wrong radio map to the current environment. That is, the adaptive localization can achieve a good positioning accuracy of 2.13-2.81 meters, while the non-adaptive method has a poor positioning accuracy of $4.59-7.17$ meters.

Table 2: Impact of open/closed doors on average accuracy

\begin{tabular}{|c|c|c|}
\hline $\begin{array}{c}\text { Average } \\
\text { accuracy }\end{array}$ & $\begin{array}{c}\text { Close-all-doors } \\
\text { radio map }\end{array}$ & $\begin{array}{c}\text { Open-all-doors } \\
\text { radio map }\end{array}$ \\
\hline \hline $\begin{array}{c}\text { Close-all-doors } \\
\text { environment }\end{array}$ & $2.13 \mathrm{~m}$ & $4.59 \mathrm{~m}$ \\
\hline $\begin{array}{c}\text { Open-all-doors } \\
\text { environment }\end{array}$ & $7.17 \mathrm{~m}$ & $2.81 \mathrm{~m}$ \\
\hline
\end{tabular}

\subsubsection{Impact of People Blocking}

We evaluate how well the proposed adaptive localization adjusts to people blocking while leaving the other environmental factors (close-all-doors and 40\% RH level) unchanged. The experiment consisted of the following three steps. In Step 1, the target user walked on the corridor alone so that the online calibration system can collect RSSI samples to train the non-blocking radio map. In Step 2, six people surrounded the target user and walked together, 

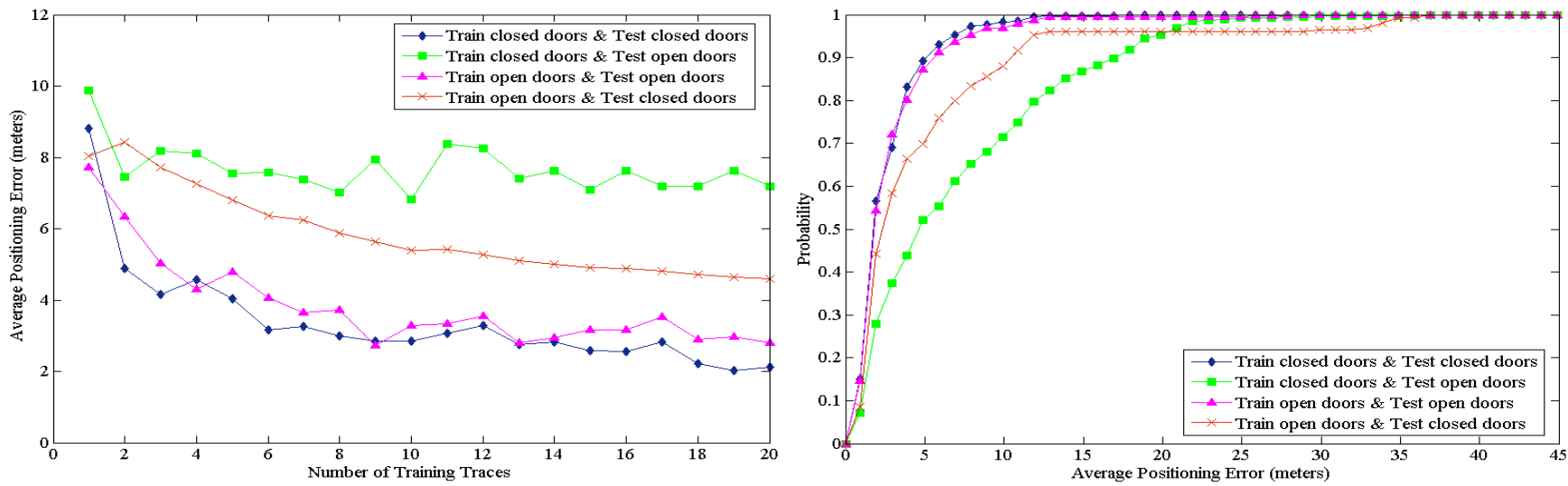

Figure 8. The left graph shows the impact of open/closed doors on the average positioning errors under different mapenvironment combinations. The right graph shows CDFs of their average positioning errors with increasing training samples.

Table 3: Impact of block-around and no-blocking people on average positioning accuracy.

\begin{tabular}{|c|c|c|}
\hline $\begin{array}{c}\text { Average } \\
\text { accuracy }\end{array}$ & $\begin{array}{c}\text { Non-blocking } \\
\text { radio map }\end{array}$ & $\begin{array}{c}\text { Block-around } \\
\text { radio map }\end{array}$ \\
\hline \hline $\begin{array}{c}\text { Non-blocking } \\
\text { environment }\end{array}$ & $2.13 \mathrm{~m}$ & $4.30 \mathrm{~m}$ \\
\hline $\begin{array}{c}\text { Block-around } \\
\text { environment }\end{array}$ & $3.96 \mathrm{~m}$ & $2.54 \mathrm{~m}$ \\
\hline
\end{tabular}

so that the online calibration can collect RSSI samples to train the block-around radio map. In Step 3, the average accuracy was measured by applying each of two radio maps (non-blocking \& block-around) to each of the two environmental conditions.

The average positioning accuracy of each map-environment combination is plotted on the left graph of Figure 9. Their cumulative distribution functions (CDF) with increasing training samples are shown on the right graph of Figure 9. Table 3 summarizes the average positioning accuracy. It leads to the conclusion that adaptive method outperforms non-adaptive method, which is similar to the results in Section 4.2.1.1.

\subsubsection{Impact of Relative Humidity}

We evaluate how well the adaptive localization adjusts to varying relative humidity $(\mathrm{RH})$ levels while leaving other environmental factors (close-all-doors and no-blocking people) unchanged. The
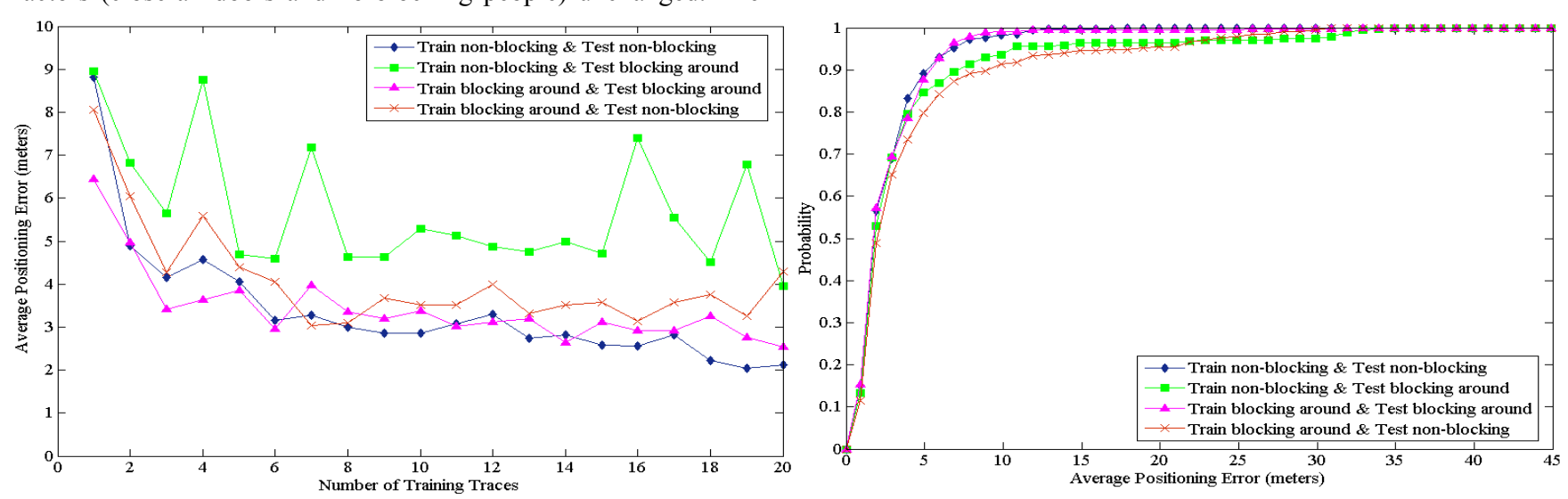

Table 4: Impact of humidity levels on average accuracy

\begin{tabular}{|c|c|c|}
\hline Average accuracy & $\begin{array}{c}40 \% \text { RH radio } \\
\text { map }\end{array}$ & $\begin{array}{c}70 \% \text { RH radio } \\
\text { map }\end{array}$ \\
\hline \hline $\begin{array}{c}40 \% \mathrm{RH} \\
\text { environment }\end{array}$ & $2.13 \mathrm{~m}$ & $3.68 \mathrm{~m}$ \\
\hline $\begin{array}{c}70 \% \mathrm{RH} \\
\text { environment }\end{array}$ & $3.06 \mathrm{~m}$ & $2.59 \mathrm{~m}$ \\
\hline
\end{tabular}

experiment consisted of the following three steps. In Step 1, a dehumidifier was run to bring the $\mathrm{RH}$ in the environment down to $40 \%$, and online calibration is then applied to train the $40 \%-\mathrm{RH}$ radio map. In Step 2, the windows were opened to allow $\mathrm{RH}$ to reached $70 \%$, and online calibration was applied to train the $70 \%$ $\mathrm{RH}$ radio map. In Step 3, the average positioning accuracy was measured by applying each of two radio maps (trained under $40 \%$ \& $70 \% \mathrm{RH}$ environments) to each of the two environmental conditions.

The average positioning accuracy of each of the four mapenvironment combinations is plotted on the left graph of Figure 10, and their cumulative distribution functions (CDF) with increasing training samples are plotted on the right graph of Figure 10. Table 4 summarizes the average positioning average. It leads to the conclusion that adaptive method outperforms non-adaptive method, which is similar to the results in Section 4.2.1.1.

igure 9. The left graph shows the impact of people on the average positioning accuracy under different map-environment combinations. The right graph shows CDFs of their average positioning errors with increasing training samples. 

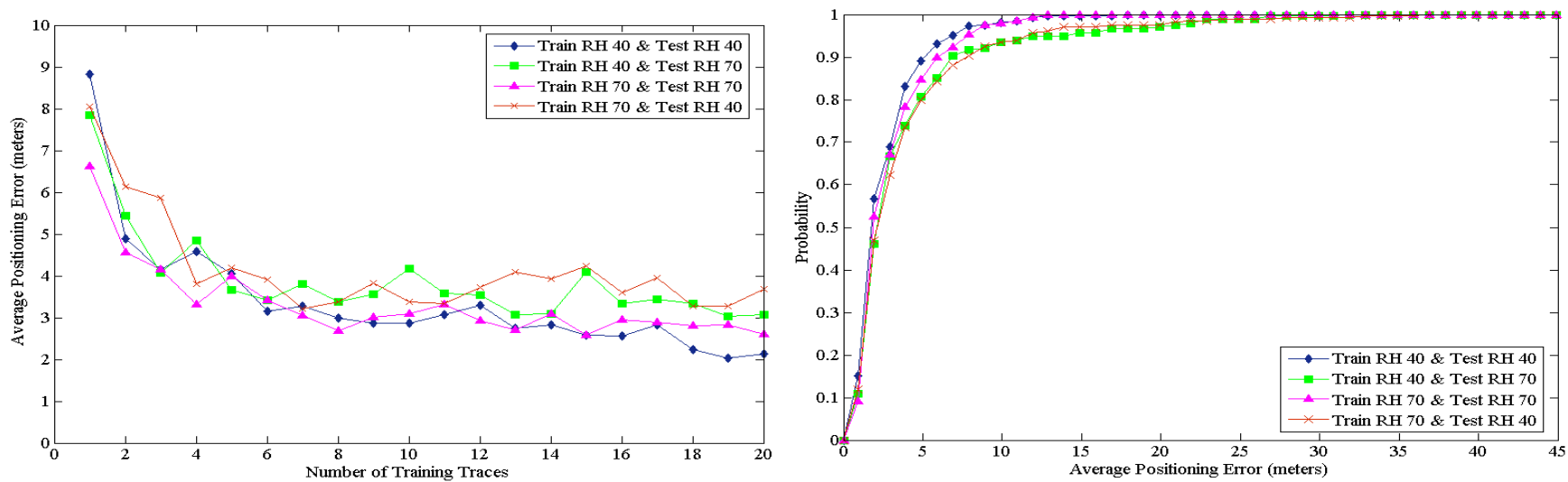

Figure 10. The left graph shows the impact of humidity (40\% and $70 \% \mathrm{RH}$ levels) on the average positioning accuracy under different map-environment combinations. The right graph shows the CDFs of their average positioning errors with increasing numbers of training samples.

\section{CONCLUSIONS AND FUTURE WORK}

This work quantitatively measured how changing environmental dynamics adversely affects the positioning accuracy in the Wi-Fi based location systems. To reduce this adverse effect, a new sensor-assisted adaptation method was proposed to adapt the localization engine to the current environmental condition. Additionally, automated online calibration was also proposed to eliminate the need for offline manual calibration. Our experimental results have shown that the error is reduced by an average of 2.6 meters in comparison to traditional non-adaptive localization methods.

The proposed systems have several limitations. Our experiments were performed mainly in the corridor, rather than within rooms. Additional RFID readers may need to be installed inside rooms to enable automatic online calibration. This requirement may add cost to the sensor part of this localization system. However, we believe that future environments will have a rich sensor infrastructure that can be shared and reused by many contextaware applications, including our localization system. For the people factor, the current study only targets the people blockaround scenario. Although block-around people can show a strong impact on the positioning accuracy, this is admittedly a rather limited case.

\section{ACKNOWLEDGMENTS}

This work was partially supported by grants from Taiwan NSC, Quanta Computer, III, and Intel (\#93-2213-E-002-088, \#93-2218E-002-146, and \#93-2622-E-002-033).

\section{REFERENCES}

[1] J. Yie, Q. Yang, L. Ni, Adaptive Temporal Radio Maps for Indoor Location Estimation. Pervasive'05, May 2005.

[2] P. Bahl and V. N. Padmanabhan. RADAR: An in-building RF-based user location and tracking system. In Proceedings of IEEE INFOCOM 2000, pages 775-784, March 2000.

[3] X. Chai, Q. Yang, Reducing Calibration Effort for Location Estimation Using Unlabeled Samples. Pervasive'05

[4] ITRI, http://www.itri.org.tw

[5] S. Willis, S. Helal, A Passive RFID Information Grid for Location and Proximity Sensing for the Blind User, University of Florida Technical Report, Oct. 2004.
[6] D. Haehnel et al., Mapping and Localization with RFID Technology. ICRA'04, pp. 1015-1020, April 2004

[7] L. M. Ni et al., LANDMARC: Indoor Location Sensing Using Active RFID. PerCom'03, March 2003.

[8] S. Y. Seidel, T. S. Rappaport, 914 MHz Path Loss Prediction Models for Indoor Wireless Communications in Multifloored Buildings. IEEE Transactions on Antennas and Propagation, 40(2):207-217, Feb. 1992.

[9] Y. Wang, X, Jia, H.K. Lee. An indoors wireless positioning system based on wireless local area network infrastructure. SatNav'03, July 2003.

[10] V. Seshadri, G. V. Zaruba, and M. Huber. A Bayesian Sampling Approach to In-door Localization of Wireless Devices Using Received Signal Strength Indication. PerCom '05, March 2005.

[11] P. Bahl, A. Balachandran, and V. Padmanabhan, Enhancements to the RADAR user location and tracking system. Technical report, Microsoft Research, February 2000.

[12] J. Hightower, and G. Borriello, Particle Filters for Location Estimation in Ubiquitous Computing: A Case Study. UbiComp '04, September 2005.

[13] Ekahau. http://www.ekahau.com

[14] Alien Technology. http://www.alientechnology.com/

[15] D. Schulz, D. Fox, and J. Hightower, People Tracking with Anonymous and ID-Sensors using Rao-Blackwellised Particle Filters, IJCAI, pp. 921-926, Aug. 2003.

[16] D. Fox, J. Hightower, L. Liao, D. Schulz, and G. Borriello, Bayesian Filtering for Location Estimation, IEEE Pervasive Computing, vol. 2, no. 3, pp. 24-33, IEEE Computer Society Press, July-September 2003.

[17] H. Hashemi. The indoor radio propagation channel. In Proceedings of the IEEE, volume 81, pages 943-968, 1993.

[18] J. Hightower, G. Borriello, Location Systems for Ubiquitous Computing, IEEE Computer, August 2001.

[19] Richard L. Knoblauch, Martin T. Pietrucha, and M. Nitzburg, Field Studies of Pedestrian Walking Speed and Start-Up Time. Transportation Research Record No. 1538, Pedestrian and Bicycle Research, published in 1996.

[20] K. Okuda, S. Yeh, C. Wu, K. Chang, and H. Chu, The GETA Sandals: A Footprint Location Tracking System. Workshop on Location- and Context-Awareness (LoCa 2005, also published as LNCS 3479: Location- and Context-Awareness), Munich, Germany, May 2005, pages 120-131. 